\title{
ACCRETION PROCESSES IN MIRA AB
}

\author{
M. Karovska, ${ }^{1}$ B. Wood ${ }^{2}$ M. Marengo, ${ }^{1}$ J. C. Raymond,${ }^{1}$ W. Hack,${ }^{3}$ and E. Guinan ${ }^{4}$
}

\section{RESUMEN}

La binaria en interacción Mira $\mathrm{AB}$ representa un laboratorio ideal para estudiar la pérdida de masa y los procesos de acreción en sistemas que contienen una estrella AGB y una estrella acreciente compacta ya que sus componentes se resolvieron y pueden ser estudiados individualmente. Presentamos resultados de nuestro estudio de larga duración de procesos de acreción en ciste sistema, los cuales incluyen observaciones recientes del HST que sugieren que la tasa de acreción sobre: Mira B es mucho menor que antes, lo cual indica una posible disrupción del disco de acreción.

\section{ABSTRACT}

The Mira AB interating binary provides an ideal laboratory for studying mass loss and accretion processes in systems contianing an $A G B$ star and a compact accretor, because its components have been resolved and can be studied individually. We present here results from our long term study of accretion processes in this system. These inchude recent HST observations suggesting that the accretion rate onto Mira B is much lower than before, indicating possible disruption of the accretion disk.

\section{key Words: ACCRETION, ACCRETION DISKS - STARS: AGB AND POST AGB - STARS: MASS LOSS}

\section{INTRODUCTION}

Mira (o Ceti, HD 14386) is the prototype for a class of pulsating stars on the asymptotic giant branch $(\mathrm{AGB})$, likely progenitors of Planetary Nebulace. Mira has a companion star, Mira B, which is located about 0.6" away (Joy 1926, Karovska et al. 1991), corresponding to a projected distance of about $70 \mathrm{AU}$ at Mlira's distance of $128 \pm 18 \mathrm{pc}$ (Perryman et al. 1997). The strong pulsation-driven wind of Mira A forms an arcretion disk around the companion. Nira $B$ is generally assumed to be a white dwarf (e. g. Reimers (- Casssatella 1985).

Mira $A B$ is one of the few wind ancreting sistems in which the components of the binary are resolvable. It is therefore a unique laboratory for studying wind accretion processes, a common but not yet understood phenomenon in many astronomical sources.

In the past two decades wo have been carrying out multi-wavelength studies of this system using various ground- and space-based facilities. Wo applied various observational techniques including high resolution imaging and interferometry, photometry. and spectroscopy at wavelengths ranging from X-ray to mid-IR to study the physical characteristics of the individual components, and the accretion phe-

\footnotetext{
${ }^{1}$ Harvard-Smithsonian Astrophysical Otsiervatory. Cambridge, USA.

${ }^{2}$ JILA, Boulder, LSA.

${ }^{3}$ STScI, Baltimore, ISA

${ }^{4}$ Villanova University, USA.
}

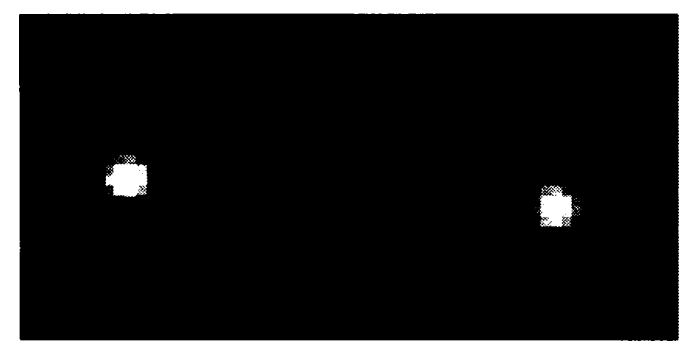

Fig. 1. HST/FOC image of Mira A (right) and its nearby hot companion Mira B (left) taken on December 11, 1995 at $5500 \AA$. The stars are separated by an angular size of only 0.6 arcseconds ( 70AU).

nomena in this system. We summarize here results from our observations of Mira $\mathrm{AB}$ and discuss recent variations in the UV spectrum of Mira B likely associated with changes in accretion processes in the system.

\section{RECENT RESULTS}

In 1995 we resolved the Mira AB system at UV and optical wavelengths using the HST/FOC camera (Fig. 1) and obtained for the first time spectra of each component (Karovska et al. 1997). The UV image of Mira A showed evidence for mass outflow from Nira $\mathrm{A}$ in the direction of the companion (Fig. 2). The small hook-like appendage extending from Mira A toward Nira B could be material being gravitationally drawn toward the accreting compact companion. 


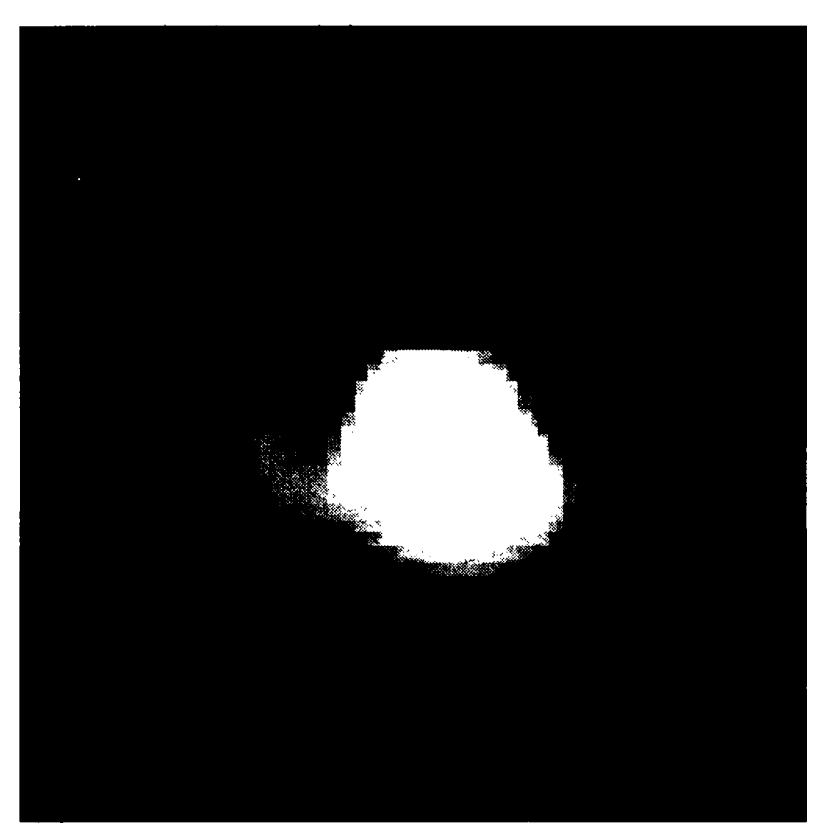

Fig. 2. First UV image of Mira A showing an outflow toward Mira B (FOV $\sim 0.15 \times 0.15$ areseconds.)

Mid-IR imaging of the Mira AB circumbinary environment carried out in 1999 using the MIRAC3 camera at the NASA Infrared Telescope Facility resolved a dusty envelope at 9.8, 11.7, and $18 \mu \mathrm{m}$ (Marengo et al. 2001). We detected strong deviations from spherical symmetry in the images of the system, including possible dust clumps in the direction of the companion (Fig. 3). These observations suggest that Mira B plays an active role in shaping the morphology of the circumstellar environment of Mira A as it evolves toward the Planetary Nebula phase.

A follow up HST/STIS spectroscopy of Mira A and Mira B carried out in July 1999 showed a dramatic change in the spectrum of Mira B. The HST/STIS spectra show an order of magnitude drop in UV emission from the HST/FOC spectra obtained in 1995 and more then an order of magnitude drop from what IUE observed a decade ago (Karovska et al. 1997; Wood, Karovska, Hack 2001).

In addition to the general fading of the accretion luminosity, another baffling development was the appearance of a forest of Ly $\alpha$-fluoresced $\mathrm{H}_{2}$ emission lines, which dominate the HST spectra despite not being seen at all in the 1995 observations or by IUE (Wood et al. 2002). A similar drop in the accretion luminosity and appearance of a set of Ly $\alpha$-fluoresced $\mathrm{H}_{2}$ emission lines (Werner band lines) were also detected in the FUSE spectra obtained two years after the HST observations (Wood \& Karovska 2004).

We analyzed the entire set of $\mathrm{H}_{2}$ lines from the HST and FUSE observations to refine estimates of
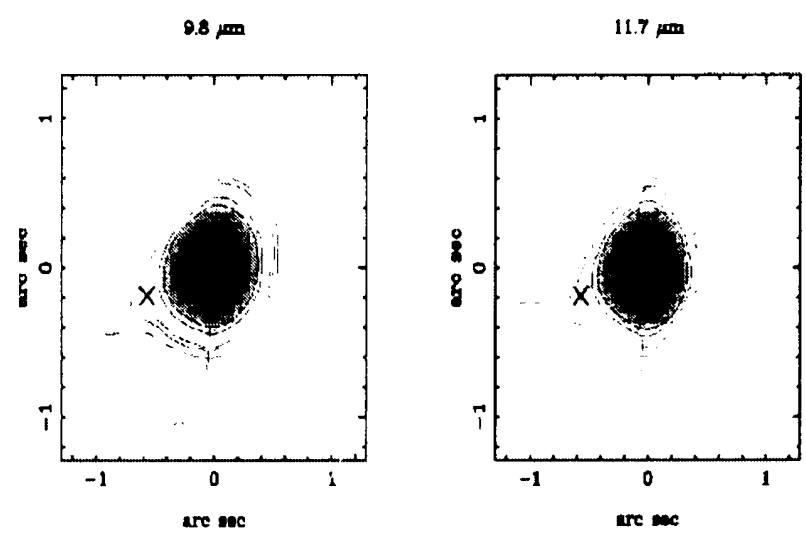

Fig. 3. Mid-IR images of Mira AB dust envelope showing an asymmetry toward Mira $\mathrm{B}$ (FOV $\sim 2 \times 2$ arcseconds.)

the physical properties of the emitting $\mathrm{H}_{2}$ gas (Wood and Karovska 2004). Our analysis shows that the emission can be reproduced by an $\mathrm{H}_{2}$ layer with a temperat,ure and column density of $\mathrm{T}=3900 \mathrm{~K}$ and $\log \mathrm{N}\left(\mathrm{H}_{2}\right)=-17.1$. respectively.

We also detected significant changes in several prominent UV lines, including in the $\mathrm{Mg}$ II $\mathrm{h} \& \mathrm{k}$ lines. Figure 4 shows the $\mathrm{Mg}$ II $\mathrm{k}$ line profile observed by HST/STIS, plotted on a velocity scalc centered on the stellar rest frame. We estimated the intrinsic line profile above the wind absorption feature (thin solid line in Fig. 4). The wind absorption was modeled to determine the mass loss rate $(\dot{M})$ and terminal velocity $\left(V_{\infty}\right)$ that yield the best fit to the data (Wood, Karovska, \& Hack 2001), shown as a thick solid line in Fig. 4.

The best fit estimate gives a mass loss rate for Mira B of $\dot{M}=5 \times 10^{-13} \mathrm{M}_{\%}, \mathrm{yr}^{-1}$ and an outflow velocity of $V_{\infty}=250 \mathrm{~km} \mathrm{~s}^{-1}$. Similar analysis and fitting were carried out using a typical MIg II k line profile observed by IUE (Fig. 3b). In this case the best fit estimates for mass loss rate and outflow velocity are respectively $\dot{M}=1 \times 10^{-11} \mathrm{M} . \mathrm{rr}^{-1}$ and $V_{\infty}=400 \mathrm{~km} \mathrm{~s}^{-1}$. The results show that the mass loss and terminal velocity of Mira $B$ 's wind were much higher a decade before the HST observations.

\section{DISCUSSION}

Our current interpretation of the $\mathrm{H}_{2}$ lines is that they originate within Mira A's massive wind, even perhaps in the region of interaction between Miral $A$ and Mira $B$ winds. The $\mathrm{H}_{2}$ is being heated and photodissociated by Mira B's Ly $\alpha$ e'mission as the $\mathrm{H}_{2}$ molecules approach Mira B on their way to being accreted (Wood and Karovska 2004). Since $\mathrm{H}_{2}$ is likely the dominant constituent of Mira $A$ s wind by mass, the Ly $\alpha$ fluorescence heating and dissociation of $\mathrm{H}_{2}$ may be an important step in the accretion pro- 


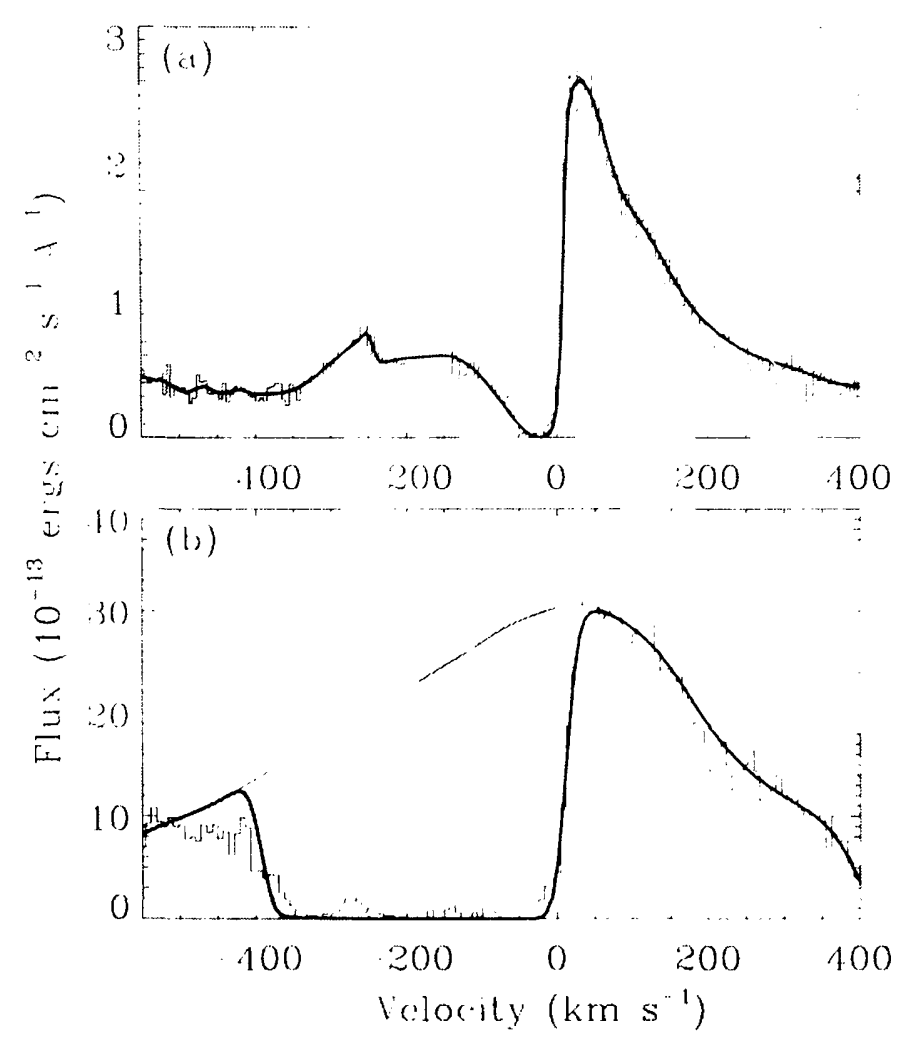

Fig. 1. (a) The $\mathrm{Vg}$ ll $\mathrm{k}$ line profile observed by HST/STIS, plotted on a velocity scale centered on the stellar rest frame. (b) A typical $\mathrm{Mg}$ II $\mathrm{k}$ line profile observed by IUE a clecalde carlier

cess, and the $\mathrm{H}_{2}$ lines are therefore useful accretion diagnostics.

We interpret the dramatic change in UV emission as a result of a decreased accretion rate onto Mira B (by all least an order of magnitude). This decrease is likely due to a disrupted accretion disk around the accretor.

This is in agreconent with the analysis of wind absorption in the $\mathrm{Mg}$ II $\mathrm{h} \& \mathrm{k}$ lines which shows that the aceretion-driven mass loss rate from Nira B at the time of the HST/STIS observations is about 20 times lower than what it was during the IUE era. consistent with a substantial decrease in accretion rate.

The drop in the UV flux observed with the HST and later with FUSE may be associated with the long-term variability of Mira $B$, and understanding the cause requires further multi-wavelength observations.

Support for this work was provided by NASA through grant numbers GO-5822.01-94A and GO08298.01-99A from the Space Telescope Science Institute, which is operated by AURA, Inc., under NASA contract NAS5-26555. We wish to thank AAVSO observers and Janet Mattei for providing the light curve of Mira A and calculation of the pulsation cycle phases. M. K. is a member of the Chandra $\mathrm{X}$-ray Center, which is operated by the Smithsonian Astrophysical Observatory under contract to NASA NAS8-39073.

\section{REFERENCES}

Joy, A. H. 1926, ApJ, 63, 333

Karovska, M., Nisenson, P., Papaliolios, C., \& Boyle, R. P. 1991, ApJ, 374, L51

Karovska, M., Hack, W., Raymond, J., \& Guinan, E. 1997, ApJ, 482, L175

Marengo, M., Karovska, M., Fazio, G. G., Hora, J. L., Hoffmann, W. F., Dayal, A., \& Deutsch, L. K. 2001, ApJ, 556, L47

Perryman, M. A. C.. et al. 1997, A\&A, 323, L49

Reimers, D., \& Cassatella, A. 1985, ApJ, 297, 275

Wood, B. E., Karrorskia. M.. \& Hack, W. 2001, ApJ, 556, $\mathrm{L} 51$

Hood. B. E., Karovska, M., \& Raymond, J.. W. 2002. ApJ.575, 1057.

Wood. B. E., Karovska, M., 2004, ApJ, in press.

M. Karovska: Harvard-Smithsonian Conter for Lstrophysics. 60 Garden Street. Cambridge, MA 02138, (karosisal refa.harvard.colu). 